\title{
Fast and Exact Mining of Probabilistic Data Streams
}

\author{
Reza Akbarinia and Florent Masseglia \\ Zenith team (INRIA-UM2), LIRMM, Montpellier, France \\ \{reza.akbarinia, florent.masseglia\}@inria.fr
}

\begin{abstract}
Discovering Probabilistic Frequent Itemsets (PFI) is very challenging since algorithms designed for deterministic data are not applicable in probabilistic data. The problem is even more difficult for probabilistic data streams where massive frequent updates need to be taken into account while respecting data stream constraints. In this paper, we propose FEMP (Fast and Exact Mining of Probabilistic data streams), the first solution for exact PFI mining in data streams with sliding windows. FEMP allows updating the frequentness probability of an itemset whenever a transaction is added or removed from the observation window. Using these update operations, we are able to extract PFI in sliding windows with very low response times. Furthermore, our method is exact, meaning that we are able to discover the exact probabilistic frequentness distribution function for any monitored itemset, at any time. We implemented FEMP and conducted an extensive experimental evaluation over synthetic and real-world data sets; the results illustrate its very good performance.
\end{abstract}

Keywords: Probabilistic Data Streams, Probabilistic Frequent Itemsets, Sliding Windows.

\section{Introduction}

Dealing with probabilistic data has gained increasing attention these past few years in both static and streaming data management and mining [3], [9], [2], [1], 10]. There are many possible reasons for probabilistic data, such as noise occurring when data are collected, noise injected for privacy reasons, semantics of the results of a search engine (often ambiguous), etc. Thus, many sensitive domains now involve massive probabilistic data. Example 1 illustrates a collection of probabilistic data, where each record is associated to a probability of occurrence.

Example 1. Let us consider animals' health monitoring in a zoo, and more particularly the health of Pandas, for which reproduction is an important issue. In our scenario, a set of body sensors gathers physiological data (blood pressure, temperature, etc.) and transforms it into possible activities thanks to a given model. For instance, the rule $\{$ pressure $=[100 . .150]$, temperature $=[80 . .90] \rightarrow$ 
sleeping, $75 \%$ means that with a blood pressure between 100 and $150 \mathrm{mmHg}$, and a body temperature between 80 and 90 Fahrenheit, the probability that a Panda is sleeping is $75 \%$. Figure 1 illustrates the activities inferred for the pandas. We can observe, for instance, that Yuan Zi was eating at 9am, with a probability of $40 \%$.

With the probabilistic approach illustrated by Example 1, there are two cases for each probabilistic record: either it really occurred in the real world or it did not. A reliable framework for handling such probabilistic data lies in the theory of "possible worlds" 6] where each unique combination of records' existence corresponds to a possible world. Unfortunately, there is a combinatorial explosion in the number of possible worlds ( $n$ records, each associated to 2 possible values of existence, leading to $2^{n}$ possible worlds). Therefore, in this context, frequent itemset mining [1] must be carefully adapted. Finding the number of occurrences of an itemset $X$ in a database $D$ (also called the support of $X$ in $D$ ) is at the core of frequent itemset mining. In the literature, we find two main support measures for probabilistic data: Expected Support [5] (an approximate measure of support) and Probabilistic Support 3. (that is an exact measure of support in probabilistic data). We work with Probabilistic, and we propose a solution for Probabilistic Frequent Itemset (PFI) mining in data streams using this measure of interest.

\begin{tabular}{|c|c|c|c|c|c|c|c|}
\hline \multicolumn{4}{|c|}{ Huan Huan } & \multicolumn{4}{|c|}{ Yuan Zi } \\
\hline e & $\mathbf{h}$ & activity & Prob. & e & h & activity & Prob. \\
\hline 1 & 8 & sleeping & 0.3 & 2 & 8 & sleeping & 0.9 \\
\hline 3 & 9 & eating & 0.3 & 4 & 9 & eating & $\overline{0.4}$ \\
\hline 5 & $\overline{10}$ & sleeping & 0.3 & 6 & 10 & drinking & 1 \\
\hline 7 & 11 & grooming & 0.4 & 8 & 11 & grooming & 0.9 \\
\hline 9 & $\overline{12}$ & sleeping & 0.3 & $\overline{10}$ & 12 & marking & 0.4 \\
\hline$\overline{11}$ & 13 & drinking & 0.3 & $\overline{12}$ & 13 & resting & 0.2 \\
\hline$\overline{13}$ & $\overline{14}$ & courting & 0.9 & $\overline{14}$ & 14 & climbing & 0.2 \\
\hline 15 & 15 & resting & 0.2 & 16 & 15 & courting & 0.4 \\
\hline 17 & 16 & playing & 0.4 & 18 & 16 & playing & 0.3 \\
\hline 19 & 17 & growling & 0.2 & 20 & 17 & growling & 0.9 \\
\hline
\end{tabular}

Fig. 1. Panda's activities inferred from body sensor data

There are several ways to observe a data stream, two important ones being batches and sliding windows [8]. Both techniques have pros and cons. Batches allow fast processing but the result is available only after the batch has been fulfilled (which is not compatible with real time constraints). Sliding windows allow maintaining the result any time the stream is updated, but they need more CPU. Today, existing methods for probabilistic data stream mining are batchbased and work with Expected Support [17, 11, 10. Meanwhile, working with sliding windows is a major matter for numerous monitoring applications where handling "anytime queries" is crucial. Data stream mining over a sliding window requires to provide efficient solutions for updating probabilistic supports after adding/removing transactions and, in the probabilistic context, this is quite challenging. 
In this paper, we introduce FEMP (Fast and Exact Mining of Probabilistic data streams), a framework adopting the exact approach while meeting the time limitations of data stream environments. To the best of our knowledge, FEMP is the first solution for PFI mining in a sliding window over probabilistic data streams.

Our contributions are i) a new model for probabilistic data streams, where an item may have multiple occurrences (each associated to a probability) for one transaction ii) a new approach for computing probabilistic support by recursion on the transactions (this approach allows to develop efficient algorithms for updating probabilistic support after any modification in the sliding window) and iii) new algorithms for probabilistic frequent itemset mining with sliding windows, where transactions are inserted or deleted. Our algorithms allow updating the new probabilistic support of any monitored itemset with a low complexity since they avoid scanning the whole sliding window from scratch.

We validated our solution through experimentation over synthetic and realworld data sets. The results show that it is able to discover and manage PFI in data streams with response times that are up to several orders of magnitude faster than baseline methods of the literature employed in a sliding window context.

\section{Problem Definition}

We now describe the problem we address with formal definitions of the probabilistic model we adopt, probabilistic itemset mining and probabilistic data streams.

\subsection{Probabilistic Data}

Let $I$ be a set of literals. $I$ is also called the vocabulary. An event $e_{i}$ is a tuple $e_{i}=\langle$ Oid,ts, $x, P>$ where $i$ is the identifier of the event, Oid is an object identifier, $t s$ is a timestamp, $x \in I$ is an item and $P$ is an existential probability $P \in[0,1]$ denoting the probability that $e_{i}$ occurs.

Example 2. Consider the data given by Figure 1, the first two events for Huan Huan are: $e_{1}=<$ Huan Huan, 8, sleeping, $0.3>$ and $e_{3}=<$ Huan Huan, 9, eating, $0.3>$.

Definition 1. A probabilistic item $x$ is an item that appears in an event, the probability of $x$ is the probability of its event.

Definition 2. A probabilistic transaction $t$ is a set of pairs $(x, P)$ for an object such that $x$ is a probabilistic item and $P$ is the probability of the event of $x$. $P(x \in t)$ is the probability of existence of $x$ in $t$. A probabilistic database is a set of probabilistic transactions. 


\begin{tabular}{|c|c|c|}
\hline Panda & Id & Transaction \\
\hline Huan Huan & $t_{1}$ & (eating, 0.3); (sleeping, 0.3) \\
\hline Yuan Zi & $t_{2}$ & (eating, 0.4); (drinking, 1) \\
\hline
\end{tabular}

Fig. 2. The pandas' activities (probabilistic transactions) from 9am to 10am

Example 3. Figure 2 gives the probabilistic transaction database of Huan Huan and Yuan Zi for two hours, from 9am to 10am. We can observe that Yuan Zi's activities in this time window were: eating with a probability of $40 \%$ and drinking with a probability of $100 \%$.

\begin{tabular}{|c|c|c|}
\hline & Possible Worlds & Probability \\
\hline$w_{1}$ & \{\}$;\{$ drinking $\}$ & 0.294 \\
\hline$w_{2}$ & $\{$ eating\}; $\{$ drinking $\}$ & 0.126 \\
\hline$w_{3}$ & $\{$ sleeping\}; $\{$ drinking $\}$ & 0.126 \\
\hline$w_{4}$ & $\{$ eating, sleeping $\} ;\{$ drinking $\}$ & 0.054 \\
\hline$w_{5}$ & \{\}$;\{$ eating, drinking $\}$ & 0.196 \\
\hline$w_{6}$ & $\{$ eating $\} ;\{$ eating, drinking $\}$ & 0.084 \\
\hline$w_{7}$ & $\{$ sleeping $; ;$ eating, drinking $\}$ & 0.084 \\
\hline$w_{8}$ & $\{$ eating, sleeping\}; eating, drinking $\}$ & 0.036 \\
\hline
\end{tabular}

Fig. 3. Possible worlds for the database illustrated in Figure 2

A probabilistic database can be treated as a set of deterministic databases, called possible worlds. The possible worlds are generated from the possible instances of transactions. Let $w$ be a possible world, then the instance of a transaction $t$ in $w$ is denoted by $t_{w}$. Figure 3 shows the possible worlds for the database in Figure 2. In this database, the instance of transaction $t_{1}$ in $w_{3}$ is $\{$ sleeping $\}$, and that of transaction $t_{2}$ is $\{$ drinking $\}$. For each possible world $w$, there is probability $P(w)$ that is computed based on the probability of its transaction instances. The sum of the probabilities of all possible worlds of a database is equal to one. In the case of independence of events, the probability of a given world is computed as $P(w)=\prod_{t \in I} P\left(t_{w}\right)$, where $P\left(t_{w}\right)$ is the probability of $t$ 's instance in $w . P\left(t_{w}\right)$ is computed as follows: $P\left(t_{w}\right)=\left(\prod_{x \in t_{w}}(P(x \in t)) \times\left(\prod_{x \notin t_{w}}(1-P(x \in t))\right)\right.$.

Intuitively, we multiply the existential probability of $t$ items that are present in $t_{w}$ by the probability of absence of those that are not present in $t_{w}$.

Example 4. In the possible worlds shown in Figure 3. the probability of $w_{4}$ is equal to the occurrence of eating and sleeping for transaction $t_{1}$, drinking for $t_{2}$, and the non-occurrence of eating for $t_{2}$. Thus $P\left(w_{4}\right)=(0.3 \times 0.3 \times 1) \times(1-0.4)=$ 0.054 .

\subsection{Probabilistic Frequent Itemsets}

The problem of frequent itemset mining from a set of transactions $T$, as defined in [1, aims at extracting the itemsets that occur in a sufficient number of 
transactions in $T$. This is based on the number of transactions in $T$ where an itemset $X$ appears (i.e. the support of $X$ in $T$ ). In the deterministic context, computing this support is straightforward (with a scan over $T$ ). In probabilistic databases, however, the support varies from one possible world to another. For this reason, the support of an itemset in a probabilistic database, introduced in [3], is given as a probability distribution function. In other words, each possible value $i \in\{0, \ldots,|T|\}$ for the support of $X$ is associated to a probability that is the probability that $X$ has this support in the probabilistic database. Definition 3 gives a more formal definition of this notion.

Definition 3. Let $W$ be the set of possible worlds and $S_{X, w}$ be the support of $X \in I$ in world $w \in W$. The probability $P_{X, T}(i)$ that $X$ has support $i$ in the set of probabilistic transactions $T$ is given by: $P_{X, T}(i)=\sum_{w \in W, S_{X, w}=i} P(w)$. In other words, $P_{X, T}(i)$ is the cumulative probability of all possible worlds in which support of $X$ is $i$. The probability distribution function $P_{X, T}(i)$ for $i \in[0 . .|T|]$ is called the probabilistic support of $X$.

Example 5. In the possible worlds given by Figure [3, we have $P_{\text {eating,T }}(1)=$ $P\left(w_{2}\right)+P\left(w_{4}\right)+P\left(w_{5}\right)+P\left(w_{7}\right)=0.46$. In other words, the probability that exactly one Panda is eating between $9 \mathrm{am}$ and $10 \mathrm{am}$ is $46 \%$.

Definition 4. Given a support value $i$, the probability $P_{\geq X, T}(i)$ that an itemset $X$ has at least $i$ occurrences in $T$, is given by: $P_{\geq X, T}(i)=\sum_{j=i}^{|T|} P_{X, T}(j)$. Given minSup and minProb, a user minimum support and minimum probability, and $T$ a set of probabilistic transactions, an itemset $X$ is a probabilistic frequent itemset $(P F I)$ iff $P_{\geq X, D}($ minSup $) \geq \operatorname{minProb.} P_{\geq X, D}(\min S u p)$ is also called the frequentness probability of $X$.

For example, the probability that "eating" has support of at least 1 is given by $P_{\text {eating }, T}(1)+P_{\text {eating }, T}(2)=0.46+0.12=0.58$. In other words, the probability that at least one panda was eating between $9 \mathrm{am}$ and $10 \mathrm{am}$ is $58 \%$.

\subsection{Probabilistic Data Stream Mining}

In many applications, the data production rate is so high that their analysis in real time with traditional methods is impossible. Sensor networks, Web usage data, scientific instruments or bio-informatics, to name a few, have added to this situation. Because of their rate, data streams should often be observed through a limited observation window and their analysis is highly constrained (e.g. "in real-time", "with ongoing queries", "with no access to outdated data", etc.). There are several models for this observation, including sliding windows [16. Definition 5 gives a formal definition of this notion.

Definition 5. An event data stream (or data stream) is an unbounded stream of ordered events. Given n, the maximum number of events to maintain in memory, a sliding window over a data stream contains the last $n$ events from the stream. 
The problem of probabilistic frequent itemset mining in a sliding window is to extract the set of probabilistic frequent itemsets after each update. The updates occur when a new event is added to the stream and the oldest one is removed from the sliding window.

\section{$3 \quad$ PFI Mining in Sliding Windows}

We now introduce FEMP, our framework for PFI mining in probabilistic data streams with a sliding window $S W$. FEMP allows monitoring the probabilistic support of all the itemsets of $S W$ in real time, as opposed to the batch model where these results are obtained only when a batch is complete. However, the main challenge in this approach consists in updating the probabilistic support of an itemset $X$ when a transaction $t$ is added to, or removed from the sliding window. In deterministic data, this operation is simple, we just check if $X \subseteq t$ and update its support consequently. In the context of possible worlds, there is no such straightforward approach, because the set of possible worlds changes completely, after adding/removing a transaction to/from the sliding window.

Before describing our solution, we mention that one of its requirements is to know $P(X \subseteq t)$, the probability that itemset $X$ is included in transaction $t$. In the case of independent items, it can be computed as $P(X \subseteq t)=\prod_{i=1}^{|X|} P\left(x_{i} \in t\right)$. In the case where items of transaction $t$ are dependent, for computing $P(X \subseteq t)$ we have to take into account the rules defined on the dependency of items. For example, if two items $x_{1}$ and $x_{2}$ have a mutual exclusion dependency, then the probability that $X=\left\{x_{1}, x_{2}, \ldots\right\}$ is a subset of a transaction $t$ is zero.

\subsection{Sliding Window Model}

Our sliding window model maintains a set of probabilistic transactions in memory. When the stream produces a new event $e_{i}=\langle$ Oid, time, $x, P\rangle$, the corresponding object in the model is either created or updated in the window. With streaming data, an item $x$ may occur at several points in time and each occurrence is associated to a probability. Therefore, we must give a reliable probability

\begin{tabular}{|c|c|}
\hline Panda & Sliding window of size 6 , after $e_{8}$ \\
\hline Huan Huan & (eating, 0.3); (sleeping, 0.3); (grooming, 0.4) \\
\hline \begin{tabular}{l|l} 
Yuan $\mathrm{Zi}$ &
\end{tabular} & (eating, 0.4); (drinking, 1); (grooming, 0.9) \\
\hline & Sliding window of size $\mathbf{6}$, after $e_{9}$ \\
\hline Huan Huan & (eating, 0.3); (sleeping, 0.51); (grooming, 0.4) \\
\hline Yuan Zi & (eating, 0.4); (drinking, 1); (grooming, 0.9) \\
\hline & Sliding window of size 6 , after $e_{10}$ \\
\hline Huan Huan & (sleeping, 0.51); (grooming, 0.4) \\
\hline \begin{tabular}{|l|l|} 
Yuan $\mathrm{Zi}$ \\
\end{tabular} & (eating, 0.4); (drinking, 1); (grooming, 0.9); (marking, 0.4) \\
\hline
\end{tabular}

Fig. 4. Sliding windows of size 6 from $e_{3}$ to $e_{10}$ 
of existence of $x$, by taking each probability of occurrence into account. To that end, we consider $P(x \in t)$ as the probability that at least one occurrence of $x$ exists in $t$ (i.e. 1 minus the probability that $x$ does not exist in $t$ ).

Example 6. Consider the stream of events illustrated in Figure 1 and $S W$, the sliding window limited to the last 6 events. Figure 4 illustrates the content of $S W$ from 11 am (i.e. $e_{3}$ to $e_{8}$ ) to $12 \mathrm{am}$ (i.e. $e_{5}$ to $e_{10}$ ). In this example, when $e_{9}$ is added, we update the probability of sleeping for Huan Huan, but we do not need to remove any item from $S W$. Then, after $e_{10}$, we add marking to the probabilistic transaction of Yuan $\mathrm{Zi}$ and $e_{3}$, the oldest event, must be removed.

\subsection{Computing Frequentness Probability}

For computing the probability that an itemset $X$ is frequent, we need to sum up the probabilities of all supports $i$ for $i>$ minsup. In other words, we have $P_{\geq X, T}($ minsup $)=\sum_{i=\text { minsup }}^{|T|} P_{X, T}(i)$, where $P_{X, T}(i)$ is the probability of support $i$ for $X$ in $T$. Notice that the sum of the probabilities in each row is equal to one. Therefore, we have: $P_{\geq X, T}($ minsup $)=\left(1-\sum_{i=0}^{\text {minsup }-1} P_{X, T}(i)\right)$. We use this equation for computing the frequentness probability of itemsets. To update the frequentness probabilities after inserting/deleting a transaction, we need to compute and update the probability of support $i(0 \leq i \leq \operatorname{minSup}-1)$ for an itemset $X$ after inserting/deleting a transaction to/from the sliding window. Our approach for computing the probabilistic support of itemsets uses a recursion on transactions.

\subsection{Recursion on Transactions}

Let $X$ be an itemset, $D B^{n}$ be a probabilistic database involving transactions $T=\left\{t_{1}, \ldots, t_{n}\right\}$, and $P_{X, T}(i)$ be the probability that the support of $X$, in the set of transactions $T$, is $i$. We develop an approach for computing $P_{X, T}(i)$ by doing recursion on the number of transactions.

Base. Let us first consider the recursion base. Consider $D B^{1}$ be a database that involves only transaction $t_{1}$. In this database, the support of $X$ can be zero or one. The support of $X$ in $D B^{1}$ is 1 with probability $P\left(X \subseteq t_{1}\right)$, and its support is 0 with probability $\left(1-P\left(X \subseteq t_{1}\right)\right)$. Thus, for the probabilistic support of $X$ in $D B^{1}$, we have the following formula:

$$
P_{X,\left\{t_{1}\right\}}(i)= \begin{cases}P\left(X \subseteq t_{1}\right) & \text { for } \mathrm{i}=1 \\ \left(1-P\left(X \subseteq t_{1}\right)\right) & \text { for } \mathrm{i}=0 \\ 0 & \text { otherwise }\end{cases}
$$

Recursion Step. Assume we have $D B^{n-1}$, a database involving the transactions $t_{1}, \ldots, t_{n-1}$. We construct $D B^{n}$ by adding transaction $t_{n}$ to $D B^{n-1}$. If $X \nsubseteq t_{n}$ then the probability of support $i$ for $X$ in $D B^{n}$ is exactly the same as that in $D B^{n-1}$. If $X \subseteq t_{n}$ then two cases can lead to a support of $i$ for $X$ in $D B^{n}$ : 
1. $X \subseteq t_{n}$ in $D B^{n}$ and the support of $X$ in $D B^{n-1}$ is equal to $i-1$. Thus, we have:

$$
P_{X, T}(i)=P_{X, T-\left\{t_{n}\right\}}(i-1) \times\left(P\left(X \subseteq t_{n}\right)\right) .
$$

2. $X \nsubseteq t_{n}$ and the support of $X$ in $D B^{n-1}$ is equal to $i$. Thus, we have:

$$
P_{X, T}(i)=P_{X, T-\left\{t_{n}\right\}}(i) \times\left(1-P\left(X \subseteq t_{n}\right)\right) .
$$

Then, the probability of support $i$ for $X$ in a database containing $t_{1}, \ldots, t_{n}$ is computed based on theorem 1 .

Theorem 1. Given an itemset $X$ and a set of transactions $T=\left\{t_{1}, \ldots, t_{n-1}, t_{n}\right\}$, the probabilistic support of $X$ in $T$ can be computed based on the probabilistic support in $T-\left\{t_{n}\right\}$ by using the following equation:

$$
\begin{aligned}
P_{X, T}(i) & =P_{X, T-\left\{t_{n}\right\}}(i-1) \times\left(P\left(X \subseteq t_{n}\right)\right) \\
& +P_{X, T-\left\{t_{n}\right\}}(i) \times\left(1-P\left(X \subseteq t_{n}\right)\right)
\end{aligned}
$$

Proof. Implied by the above discussion.

\subsection{Updating Probabilistic Support after Inserting a Transaction}

To efficiently support data mining over probabilistic data streams, we need to update efficiently the probabilistic support of itemsets after each update. Here, we deal with the insertion of a new transaction to the sliding window. The case of transaction removal will be addressed in Section 3.5

After inserting a new transaction to the sliding window, the probabilistic support can be updated by an algorithm that proceeds as follows (we removed the pseudo-code due to space restrictions). Let $P_{X, T}[0 . .|S W|]$ be an array such that $P_{X, T}[i]$ shows the probability of support $i$ for itemset $X$ in a set of transactions $T$. $|S W|$ is the maximum support of a transaction in the sliding window, i.e. the size of the window. Given $P_{X, T}$, we generate an array $P_{X, T+\{t\}}$ such that $P_{X, T+\{t\}}[i]$ shows the probability of support $i$ for $X$ in $T+\{t\}$. For filling the array $P_{X, T+\{t\}}$, our algorithm considers two main cases: either $T$ is empty or $T$ is not empty (so $P_{X, T}$ is available). In the first case, we have only one transaction in the sliding window. Thus, our algorithm initializes $P_{X, T+\{t\}}$ using the base of our recursive formula (described in Section [3.3) by setting $P_{X, T+\{t\}}[1]=P(X \subseteq t)$ and $P_{X, T+\{t\}}[0]=1-P(X \subseteq t)$. In the second case, i.e. where $T$ is not empty, the algorithm computes the values of $P_{X, T+\{t\}}$ based on those in $P_{X, T}$ by using our recursive formula (i.e. Equation 2) as follows:

$$
P_{X, T+\{t\}}[i]=\left(P_{X, T}[i-1] \times P(X \subseteq t)\right)+\left(P_{X, T}[i] \times(1-P(X \subseteq t))\right)
$$

When $P(X \subseteq t)=0$ we can simply ignore the transaction since it has no impact on the support, thus we have $P_{X, T+\{t\}}=P_{X, T}$. Recall that for computing the frequentness probability of itemsets, we need to know only the probability of supports between zero and minSup - 1. This is the reason why in our algorithm we fill the array only for the values that are lower than minSup. Example 7 illustrates our algorithm. 
Example 7. Figure 5 shows the execution of our algorithm over the database shown in Figure2, with $\mathrm{X}=$ eating. Recall that, in this database, we have: $P(X \subseteq$ $\left.t_{1}\right)=0.3$ and $P\left(X \subseteq t_{2}\right)=0.4$. Initially $T=\{\}$, then we add $t_{1}$ and afterwards $t_{2}$ to it. In the fist row, the algorithm sets the probabilistic supports for $T=\left\{t_{1}\right\}$. Thus, we have $P_{X, T+\left\{t_{1}\right\}}[1]=P\left(X \subseteq t_{1}\right)=0.3$ and $P_{X, T+\left\{t_{1}\right\}}[0]=(1-P(X \subseteq$ $\left.\left.t_{1}\right)\right)=1-0.3=0.7$. The probabilities in the second row are computed using our recursive formula. For example, $P_{X,\left\{t_{1}, t_{2}\right\}}[1]=\left(P_{X,\left\{t_{1}\right\}}[0] \times P\left(X \subseteq t_{2}\right)\right)+$ $\left(P_{X,\left\{t_{1}\right\}}[1] \times\left(1-P\left(X \subseteq t_{2}\right)\right)=(0.7 \times 0.4)+(0.3 \times 0.6)=0.46\right.$.

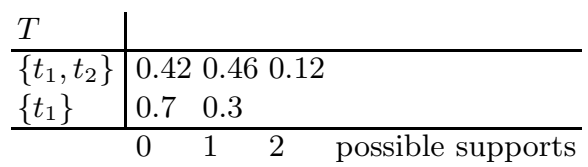

Fig. 5. Computing the probabilistic support of eating in the probabilistic database of Figure 2

The time complexity of our algorithm for updating the probabilistic support of an itemset $X$ after inserting a new transaction to the sliding window is $O$ (minsup). Its space complexity is $O(|S W|)$ where $|S W|$ is the size of the sliding window, i.e. the maximum number of transactions in the window.

\subsection{Updating Probabilistic Support after Deleting a Transaction}

Assume we have the probabilistic support of an itemset $X$ for a set of transactions $T$, then the question is: "how to compute the probabilistic support in $T-\{t\}$ ?" One might think that the probabilistic support $i$ for $X$ in $T-\{t\}$ (i.e. $\left.P_{X, T-\{t\}}(i)\right)$ could be computed as $P_{X, T}(i-1) / P(X \subseteq t)+P_{X, T}(i) /(1-P(X \subseteq$ $t)$ ). Unfortunately, this formula will not work. For example, if we use it for computing $P_{\text {eating },\left\{t_{1}\right\}}(1)$ after deleting transaction $t_{2}$ from the database used in Example 7 then we obtain $0.42 \times 0.4+0.46 \times 0.6=0.444$, whereas the value of $P_{\text {eating },\left\{t_{1}\right\}}(1)$ is equal to 0.3 (see Figure 5). To solve the problem of updating the probabilistic support of $X$ in $T-\{t\}$, we develop the following theorem:

Theorem 2. Let $X$ be an itemset, $T$ a set of transactions, and $P_{X, T}$ an array denoting the probabilistic support of $X$ in $T$. Assume we delete a transaction $t$ from $T$. Let $P_{X, T-\{t\}}(i)$ be the probability for $X$ to have support $i$ in $T-\{t\}$, then $P_{X, T-\{t\}}(i)$ can be computed as:

$$
\begin{aligned}
& -\frac{P_{X, T}(i)-\left(P_{X, T-\{t\}}(i-1) \times P(X \subseteq t)\right)}{1-P(X \subseteq t)} \text { if } P(X \subseteq t) \neq 1 \\
& -P_{X, T}(i+1) \text { otherwise }
\end{aligned}
$$

Proof. In the case where $P(X \subseteq t)=1$, it is obvious that by removing $t$ from $T$, the support of $X$ is reduced by one. Thus, the probability of support $i$ in 
$T-\{t\}$ is equal to the probability of support $i+1$ in $T$. For the case where $P(X \subseteq t) \neq 1$, it is sufficient to show that:

$$
P_{X, T-\{t\}}(i) \times(1-P(X \subseteq t))=P_{X, T}(i)-\left(P_{X, T-\{t\}}(i-1) \times P(X \subseteq t)\right) .
$$

For this, we expand the right side of this equation by using Equation 2 in Section 3.3 . We replace $P_{X, T}(i)$ by its equivalent, that is:

$P_{X, T-\{t\}}(i-1) \times(P(X \subseteq t))+P_{X, T-\{t\}}(i) \times(1-P(X \subseteq t))$

Thus, we have:

$P_{X, T}(i)-\left(P_{X, T-\{t\}}(i-1) \times P(X \subseteq t)\right)$

$=P_{X, T-\{t\}}(i-1) \times(P(X \subseteq t))+P_{X, T-\{t\}}(i) \times(1-P(X \subseteq t))-\left(P_{X, T-\{t\}}(i-\right.$ 1) $\times P(X \subseteq t))$

$=P_{X, T-\{t\}}(i) \times(1-P(X \subseteq t))$

Theorem 2 suggests to compute $P_{X, T-\{t\}}(i)$ based on $P_{X, T}(i)$ and $P_{X, T-\{t\}}(i-$ $1)$. To develop an algorithm based on this theorem, we need to compute $P_{X, T-\{t\}}(0)$ that is the probability of support 0 for $X$ in $T-\{t\}$. This can be done as follows. We use the fact that when a transaction $t$ is added to the sliding window, the probability of support 0 is multiplied by the probability of absence of $t$. Thus, when $t$ is removed from $T$, to compute $P_{X, T-\{t\}}(0)$ we can divide $P_{X, T}(0)$ by $(1-P(X \subseteq t))$, if $P(X \subseteq t) \neq 1$. In other words, we have:

$$
P_{X, T-\{t\}}(0)=\frac{P_{X, T}(0)}{1-P(X \subseteq t)}, \text { for } P(X \subseteq t) \neq 1
$$

Equation 3 works iff $P(X \subseteq t) \neq 1$. In the case where $P(X \subseteq t)=1$, we have $P_{X, T-\{t\}}(0)=P_{X, T}(1)$.

Based on Theorem 2 and Equation 3. we developed Algorithm 1 that updates the probabilistic support after removing a transaction from a sliding window. Recall that for finding frequent itemsets, we need only to compute the probabilistic supports for values that are lower than minSup. This is why the "for loop" in the algorithm (started at Line 10) is from 1 to $\min \{\min \operatorname{Sup}-1,|T|-1\}$. The time complexity of Algorithm 1 is $O($ minsup), and its space complexity $O(|S W|)$ where $|S W|$ is the size of the sliding window.

\section{Experiments}

We evaluate the performance of FEMP by a thorough comparison to existing algorithms in the literature that use Probabilistic Support in exact [3] and approximate 14 mining. Since we do not find sliding window approaches in the literature, we implemented these algorithms as follows: each time an event is added or removed from the sliding window, the algorithm runs, from scratch, on the content of the updated sliding window. PFIM is the algorithm of [3] implemented with all the optimizations (including the 0-1 optimization). However, due to extremely high response time in batch mode, we implemented two other versions of this algorithm. In PFIM-50\% the discovery is not performed for each event but for each two events (only $50 \%$ of the events are considered). In 


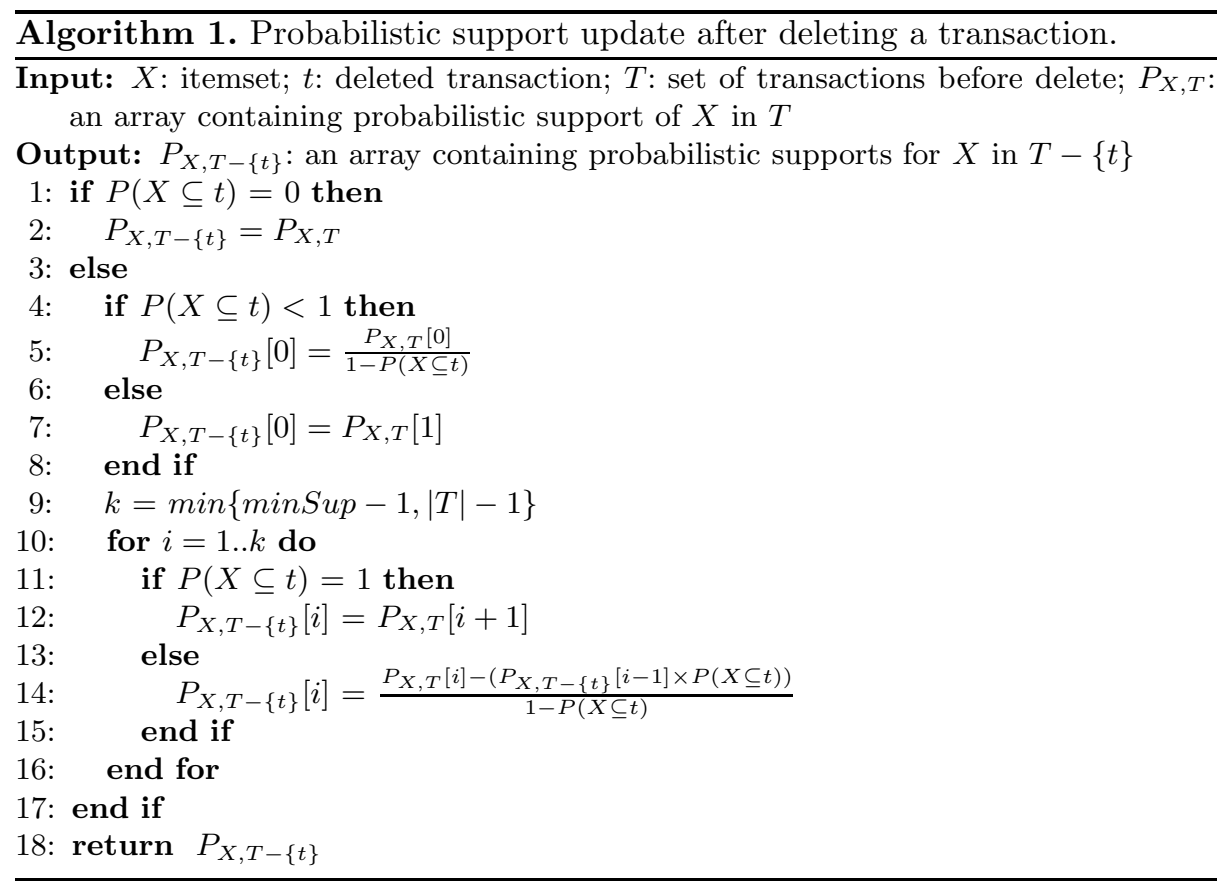

PFIM-25\%, the discovery on the sliding window is performed each 4 events. Eventually, Poisson is the algorithm of [14] (that allows approximate PFI mining) running on the whole sliding window after each update. A brief discussion on these algorithms is given in Section 5 .

We use two datasets for these experiments: a synthetic one (by the IBM 1 generator) and a real one (the "accident" dataset from the FMI repository 2 ). The synthetic dataset contains 38 millions of events, 8 millions of transactions and 100 items. The accidents dataset contains 11 millions of events, $340 \mathrm{~K}$ transactions and 468 items. We have added an existential probability $P \in] 0 . .1]$ to each event in these datasets, with a uniform distribution. For both datasets, minSup has been set to $30 \%$ of the window size and minProb to $40 \%$.

We implemented two versions of FEMP. The first one is "Dynamic-FEMP" (d-FEMP in our experiments). In this version, when a new candidate itemset is generated, it's frequentness probability will be checked over the next updates in the stream thanks to our algorithms presented in Section 3. This is the fastest approach but it implies a delay in the pattern discovery (similar to the delay described in [13]). The second version is "Exact-FEMP" (e-FEMP in our experiments). Here, each time a candidate itemset is generated it is immediately verified, from scratch, over all the transactions maintained in the current sliding window.Besides that, the probabilistic support of all existing itemsets is

\footnotetext{
${ }^{1}$ http://www.cs. loyola.edu/ cgiannel/assoc_gen.html

2 http://fimi.ua.ac.be/data/
} 
maintained at each update using our algorithms presented in Section 3 . e-FEMP guarantees an exact PFI discovery at any point in the stream. However, this is done at the price of a higher time complexity compared to d-FEMP.

We present the results obtained by these approaches on a probabilistic data streams with sliding windows. Our goal is, on the one hand, to show that a sliding window approach in probabilistic data streams with probabilistic support is possible thanks to our algorithms and, on the other hand, to illustrate the behavior of our approach in a context where transactions can be added or removed from the observation window.

\subsection{Feasibility}

Figure 6] shows the time needed by each algorithm to extract the PFI in a growing sliding window $S W$. The size of $S W$ grows from 0 to 5000 transactions for the synthetic dataset and from 0 to 10000 for the accident dataset. This corresponds to the initialization of the stream. We observe that the response time of d-FEMP increases barely since it needs very few calculations. e-FEMP increases more clearly, since it must scan $S W$ each time a new candidate is proposed. Meanwhile, all the versions of PFIM and Poisson have much higher response times. d-FEMP needs 7.34 s to fill $S W$ for the accident dataset, where PFIM needs 618s. Furthermore, we can see that Poisson is faster than all versions of PFIM after a number of transactions, but not for the first ones. This is due to the large number of infrequent patterns extracted by Poisson, caused by the approximation of Expected Support. Actually, for the first hundreds of transactions, Poisson may extract up to 146 PFI while the real number of PFI is 36 at most. Such a large number of erroneous PFI is a cause of unnecessary computations and high response times.

Figure 7 shows the time needed by each algorithm to process 100 events, while the transaction data is fed in a pass-through fashion. Although probabilistic supports are maintained after each update in the cases of d-FEMP, e-FEMP,
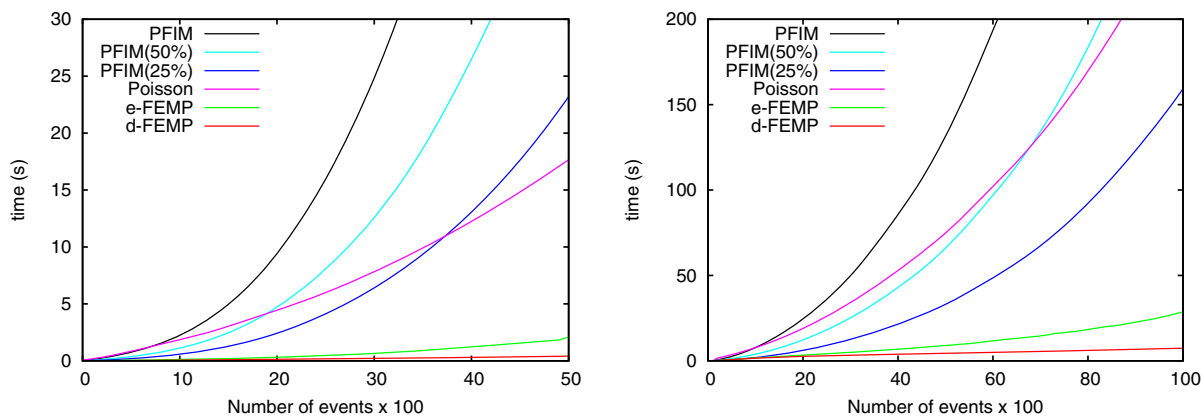

Fig. 6. Initialization time (filling $S W$ ) on synthetic (top) and accident (bottom) datasets 

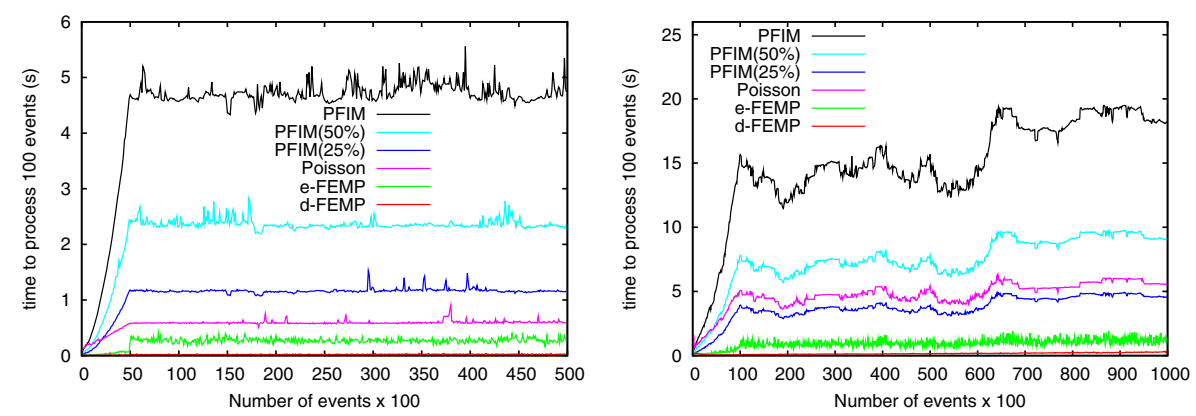

Fig. 7. Processing times for 100 events on synthetic (top) and accident (bottom) datasets

Poisson and PFIM, we report the time for 100 events because the response time of d-FEMP, for only one event, would always be 0s. That time is recorded as the number of processed events increases, from the $100^{t h}$ event to the $50000^{t h}$ one in the case of synthetic dataset $\left(100000^{t h}\right.$ for accident dataset). We observe that d-FEMP needs less than 0.05s to update the supports of the monitored itemsets in memory for each 100 updates to the stream. e-FEMP needs more time (up to $1 \mathrm{~s})$ since it has to scan $S W$ when new candidate itemsets are generated. Depending on the dataset, Poisson is faster or slower that PFIM-25\%. This is due to the difference in density between these datasets, where Poisson can extract itemsets that are not frequent (slowing down the extraction process). Over the synthetic dataset, the time needed by e-FEMP is 5 times faster than Poisson (while extracting exact probabilistic support, whereas Poisson gives an approximation with Expected Support) and up to 20 times faster than PFIM. We also observe that d-FEMP is very close to 0s. In fact, in our experimental data, d-FEMP appears to run up to two orders of magnitude faster than PFIM on the accident dataset to process 100 events. The global response time of dFEMP, as the stream passes through, is several orders of magnitude lower than that of PFIM.

\subsection{Scalability}

Figure 8 shows the execution times of each algorithm for a full sliding window. More precisely, when a sliding window $S W$ is full (after initialization), we measure the time needed to process $|S W|$ updates (one update is made of an event insertion and an event removal). This time is measured for an increasing size of $S W$. Our experiments clearly show that d-FEMP incurs very few overhead to the computations needed for maintaining the data structures. 

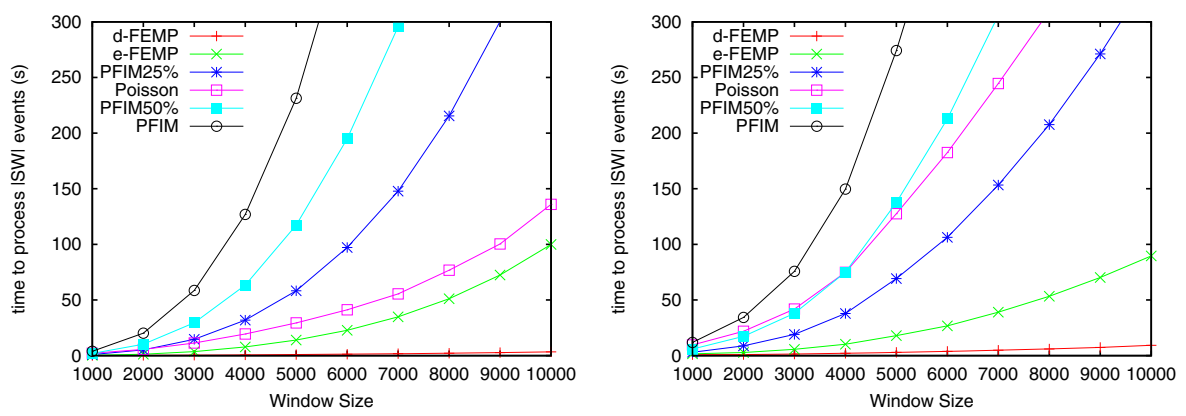

Fig. 8. Processing time for $|S W|$ updates with increasing size of $S W$ on synthetic (top) and accident (bottom) datasets

\section{Related Work}

Expected Support. Probabilistic data mining is a recent research topic that is gaining increasing attention [9], 4], [12, 10, [15. In [5], the problem of itemset mining from probabilistic data is introduced and the authors propose the notion of Expected Support as a first solution. Let $P(X \subseteq t)$ be the probability that itemset $X$ is included in transaction $t$, the Expected Support $E S(X)$ of $X$ in database $D$ is given by: $E S(X)=\sum_{j=1}^{|D|} P\left(X, t_{j}\right)$. This support is then used as a frequency measure (compared to a user minimum threshold) in U-Apriori, a level-wise approach based on the Apriori principle for frequent itemset mining.

Probabilistic Support. In [3], the authors introduce the notion of probabilistic support which is an exact measure of an itemset support in the possible world model. They define the probability that an itemset $X$ has support $i$ as in Definition 4. The authors propose to compute the frequentness probability of an itemset $X$ using a dynamic programming approach. However, their approach is incremental in the support (i.e. the transaction set is fixed and each iteration of their recursion allows computing the support probability of an itemset for an increasing support), thus not appropriate for a data stream environment.

Some approximation methods for the probabilistic support of an itemset have also been proposed. The idea of [14] is to approximate the support distribution function by means of a Poisson law. In 4, the authors propose another approximation of frequentness probability based on the central limit theorem. The main drawbacks of these approaches are to use Expected Support as a measure of probabilistic frequentness [14] and to work only on statistical independent data [14, 4].

Probabilistic Data Streams. Itemset mining in data streams is an important topic of knowledge discovery [13, [7]. Mainly, we find contributions on the extraction techniques and the data models, such as batches [7] or sliding windows [13, [16. In [8, we find a comparative study of these models. In [17], the authors 
propose to extract frequent items in probabilistic data. Their approaches allow finding items (itemsets of only one item) in static data and likely frequent items in data streams. 11 proposes to extract frequent itemset from streaming probabilistic data by means of Expected Support and a batch model. In [10, we find a batch-based approach to extract frequent itemsets using Expected Support in probabilistic data streams with a technique inspired from [7.

Despite the interest of exact PFI mining with sliding windows [8], [16], we do not find any proposal in the literature for such an approach. As we discuss in Section 3, the main challenge in this context is to update the probabilistic support of an itemset when a transaction is added to or removed from the window. Our work is therefore motivated by the needs and challenges of providing an approach that is able to i) extract PFI from data streams; ii) use sliding windows and update the support of an itemset upon transaction insertion or removal; and iii) work with statistical dependent and independent data.

\section{Conclusion}

In this paper, we proposed FEMP, the first solution for exact PFI mining in data streams with sliding windows. FEMP allows efficient computation of the exact probabilistic support of itemsets whenever a transaction is added or removed from the observation window. Compared to non-incremental algorithms, that need to scan the whole sliding window after each update, our approach shows very low execution time. Through an extensive experimental evaluation on synthetic and real datasets, we observed that FEMP can be up to several orders of magnitude faster than traditional approaches adapted to sliding windows.

\section{References}

1. Agrawal, R., Imieliński, T., Swami, A.: Mining association rules between sets of items in large databases. SIGMOD Rec. 22, 207-216 (1993)

2. Akbarinia, R., Valduriez, P., Verger, G.: Efficient Evaluation of SUM Queries Over Probabilistic Data. IEEE Transactions on Knowledge and Data Engineering (2012)

3. Bernecker, T., Kriegel, H.-P., Renz, M., Verhein, F., Zuefle, A.: Probabilistic frequent itemset mining in uncertain databases. In: Proceedings of the 15th ACM SIGKDD International Conference on Knowledge Discovery and Data Mining, KDD 2009, pp. 119-128. ACM, New York (2009)

4. Calders, T., Garboni, C., Goethals, B.: Approximation of frequentness probability of itemsets in uncertain data. In: Proceedings of the 2010 IEEE International Conference on Data Mining, ICDM 2010, pp. 749-754. IEEE, Washington, DC (2010)

5. Chui, C.-K., Kao, B., Hung, E.: Mining frequent itemsets from uncertain data. In: Zhou, Z.-H., Li, H., Yang, Q. (eds.) PAKDD 2007. LNCS (LNAI), vol. 4426, pp. 47-58. Springer, Heidelberg (2007)

6. Dalvi, N., Suciu, D.: Efficient query evaluation on probabilistic databases. The VLDB Journal 16, 523-544 (2007) 
7. Giannella, C., Han, J., Pei, J., Yan, X., Yu, P.: Mining Frequent Patterns in Data Streams at Multiple Time Granularities. In: Kargupta, H., Joshi, A., Sivakumar, K., Yesha, Y. (eds.) Next Generation Data Mining. AAAI/MIT (2003)

8. Kranen, P., Seidl, T.: Harnessing the strengths of anytime algorithms for constant data streams. Data Min. Knowl. Discov. 19, 245-260 (2009)

9. Leung, C.K.-S., Brajczuk, D.A.: Efficient algorithms for the mining of constrained frequent patterns from uncertain data. SIGKDD Explor. Newsl. 11, 123-130 (2010)

10. Leung, C.K.-S., Jiang, F.: Frequent itemset mining of uncertain data streams using the damped window model. In: Proceedings of the 2011 ACM Symposium on Applied Computing, SAC 2011, pp. 950-955. ACM, New York (2011)

11. Leung, C.-S., Hao, B.: Mining of frequent itemsets from streams of uncertain data. In: Proceedings of IEEE 25th International Conference on Data Engineering (ICDE), pp. 1663-1670 (2009)

12. Sun, L., Cheng, R., Cheung, D.W., Cheng, J.: Mining uncertain data with probabilistic guarantees. In: Proceedings of the 16th ACM SIGKDD International Conference on Knowledge Discovery and Data Mining, KDD 2010, pp. 273-282. ACM, New York (2010)

13. Teng, W.-G., Chen, M.-S., Yu, P.S.: A Regression-Based Temporal Pattern Mining Scheme for Data Streams. In: VLDB, pp. 93-104 (2003)

14. Wang, L., Cheng, R., Lee, S.D., Cheung, D.: Accelerating probabilistic frequent itemset mining: a model-based approach. In: Proceedings of the 19th ACM International Conference on Information and Knowledge Management, CIKM 2010, pp. 429-438. ACM, New York (2010)

15. Liu, Y.-H.: Mining frequent patterns from univariate uncertain data. Data and Knowledge Engineering 71(1), 47-68 (2012)

16. Zhang, C., Masseglia, F., Lechevallier, Y.: ABS: The anti bouncing model for usage data streams. In: Proceedings of the 2010 IEEE International Conference on Data Mining, ICDM 2010, pp. 1169-1174. IEEE Computer Society, Washington, DC (2010)

17. Zhang, Q., Li, F., Yi, K.: Finding frequent items in probabilistic data. In: Proceedings of the 2008 ACM SIGMOD International Conference on Management of Data, SIGMOD 2008, pp. 819-832. ACM, New York (2008) 